PROCEEDINGS OF THE

AMERICAN MATHEMATICAL SOCIETY

Volume 137, Number 1, January 2009, Pages 179-187

S 0002-9939(08)09468-9

Article electronically published on July 31,2008

\title{
BOUNDED HOLOMORPHIC FUNCTIONS WITH GIVEN MAXIMUM MODULUS ON ALL CIRCLES
}

\author{
PIOTR KOT
}

(Communicated by Mei-Chi Shaw)

\begin{abstract}
We study $\Omega \subset \mathbb{C}^{d}$, a circular, bounded, strictly convex domain with $C^{2}$ boundary. Let $g$ and $h$ be continuous functions on $\partial \Omega$ with $|g(z)|<$ $h(z)=h(\lambda z)$ for $z \in \partial \Omega$ and $|\lambda|=1$. First we prove that $h$ can be approximated by the maximum modulus values of $K$ homogeneous polynomials, where $K$ is independent from $h$. Next we construct $f_{1} \in A(\Omega)$ such that

$$
\max _{|\lambda|=1}\left|\left(g+f_{1}\right)(\lambda z)\right|=h(z)
$$

for $z \in \partial \Omega$. Moreover we can choose $f_{2} \in \mathbb{O}(\Omega)$ with $\left|f_{2}^{*}(z)\right|=h(z)$ for almost all $z \in \partial \Omega$ and $\max _{|\lambda|<1}\left|f_{2}(\lambda z)\right|=h(z)$ for all $z \in \partial \Omega$.
\end{abstract}

\section{INTRODUCTION}

Let $\Omega \subset \subset \mathbb{C}^{d}$ be a bounded, circular strictly convex domain with $C^{2}$ boundary. We study a phenomenon of the existence of a holomorphic and continuous function on $\bar{\Omega}$ with given values on the boundary. We say that a compact set $K$ is a maximum modulus set for $A(\Omega)$ if there exists $f \in A(\Omega)$ such that $|f|<1$ on $\bar{\Omega} \backslash K$ and $|f|=1$ on $K$. There is also a stronger concept of a peek set when $f=1$ on $K$.

In fact it is well known that for $d>1$ a holomorphic non-constant function $f \in A\left(\mathbb{B}^{d}\right)$ such that $|f(z)|=1$ for all $z \in \partial \mathbb{B}^{d}$ does not exist. Therefore maximum modulus sets and peak sets are extensively considered by many authors.

Topologically, peak sets and maximum modulus sets are small in strictly pseudoconvex domains, as was proved by Stout and Duchamp. The real topological dimension of a maximum modulus set is no more than $d$ [7] and for a peak set is no more than $d-1[6]$. In particular, the maximum modulus set must have an empty interior.

However, from the measure-theoretic point of view, peak sets and maximum modulus sets no longer have to be small. Stensönes Henriksen has proved [5] that every strictly pseudoconvex domain with $C^{\infty}$ boundary in $C^{d}$ has a peak set with a Hausdorff dimension $2 d-1$. However, Eric L $\varnothing \mathrm{v}$ has proved [4 that a maximum modulus set can have positive $(2 d-1)$-dimensional Hausdorff measure.

We construct a maximum modulus set which crosses any circle in $\partial \Omega$ with the center at zero. Therefore the Hausdorff dimension is at least $2 d-2$. It is also

Received by the editors September 11, 2007, and, in revised form, December 12, 2007.

2000 Mathematics Subject Classification. Primary 32A05, 32A35.

Key words and phrases. Homogeneous polynomials, maximum modulus set, inner function.

(C)2008 American Mathematical Society Reverts to public domain 28 years from publication 
possible to combine our construction with the construction of an inner function. Other constructions of inner functions can be found in [1, 3, 4].

\section{Homogeneous Polynomials}

In our constructions we use the homogeneous polynomials from the paper [2], and thus we consider the same (as in 2]) unitarily invariant pseudo-metric

$$
\rho(z, w)=\min _{|\lambda|=1}\|z-\lambda w\| .
$$

Now for $\xi \in \partial \Omega$ we define a vector $\nu_{\xi}=\frac{1}{\left\langle\xi, \overline{\eta_{\xi}}\right\rangle} \overline{\eta_{\xi}}$, where $\eta$ is a defining function of class $C^{2}$ for $\Omega$ and $\eta_{\xi}=\left(\frac{\partial \eta}{\partial z_{1}}(\xi), \ldots, \frac{\partial \eta}{\partial z_{d}}(\xi)\right)$. Recall that:

Lemma ([2, Lemma 2.1]). There exist constants $c_{1}, c_{2}>0$ such that

$$
c_{1} \rho^{2}(z, \xi) \leq 1-\left|\left\langle z, \nu_{\xi}\right\rangle\right| \leq c_{2} \rho^{2}(z, \xi)
$$

for $\xi, z \in \partial \Omega$.

Let $\alpha>0$. A subset $A \subset \mathbb{C}^{d}$ is called $\alpha$-separated if $\rho\left(z_{1}, z_{2}\right)>\alpha$ for all distinct elements $z_{1}$ and $z_{2}$ of $A$. It is clear that for $\alpha>0$ each $\alpha$-separated subset of $\partial \Omega$ is finite.

We need the following lemmas:

Lemma 2.1 ([2, Lemma 2.2]). For $z \in \partial \Omega$ let

$$
A_{k}(z):=\{\xi \in A: \alpha k t \leq \rho(z, \xi) \leq \alpha(k+1) t\} .
$$

There exists a constant $c>0$ such that if $A=\left\{\xi_{1}, \ldots, \xi_{s}\right\}$ is a $2 \alpha$-separated subset of $\partial \Omega$, then the set $A_{k}(z)$ has at most $c(k+2)^{2 d-1}$ elements. The set $A_{0}$ has at most 1 element and $s \leq c(\alpha t)^{1-2 d}$.

Lemma 2.2 ([2, Lemma 2.3]). If $A \subset \partial \Omega$ is $\alpha$ t-separated, then for each $\beta>\alpha>0$ there exists an integer $K=K(\alpha, \beta)$ such that $A$ can be partitioned into $K$ disjoint $\beta$-separated sets.

Lemma 2.3 ([2, Lemma 2.5]). Let $0<c_{1}<c_{2}$ be constants from (1). For a given $a \in(0,0.5)$ there exist constants $C>2$ and $N_{0} \in \mathbb{N}$ such that for all integers $N \geq N_{0}$, for each $C / \sqrt{c_{1} N}$-separated subset $A$ of $\partial \Omega$ and each integer $m$ with $N \leq m \leq 2 N$, the polynomial $p_{m}(z):=\sum_{\xi \in A}\left\langle z, \nu_{\xi}\right\rangle^{m}$ satisfies the following:

(1) If $z \in \partial \Omega, Q(z):=\left\{\xi \in A: \rho(z, \xi) \geq \frac{C}{2 \sqrt{c_{1} N}}\right\}$, then $\sum_{\xi \in Q(z)}\left|\left\langle z, \nu_{\xi}\right\rangle\right|^{m}<a$.

(2) If $z \in \partial \Omega$, then $A \backslash Q(z)$ has at most one element.

(3) If $\xi_{0} \in A, z \in \partial \Omega$ are such that $\rho\left(z, \xi_{0}\right) \leq \frac{a}{\sqrt{c_{2} N}}$, then

(a) $Q(z)=A \backslash\left\{\xi_{0}\right\}$,

(b) $\left|\left\langle z, \nu_{\xi_{0}}\right\rangle\right|^{m}>1-2 a^{2}$,

(c) $\left|p_{m}(z)\right|>1-2 a^{2}-a$.

(4) $\left|p_{m}(z)\right| \leq \sum_{\xi \in A}\left|\left\langle z, \nu_{\xi}\right\rangle\right|^{m}<1+$ a for all $z \in \partial \Omega$.

Now we can prove the following result:

Lemma 2.4. For a given $\theta \in(0,1)$ there exists a constant $C>2$ such that if $h$ is a continuous strictly positive function on $\partial \Omega$ with the same values on the circles, then there exists $N_{1} \in \mathbb{N}$ such that for all integers $N \geq N_{1}$, for each $\frac{C}{\sqrt{c_{1} N}}$-separated subset $A$ of $\partial \Omega$ and each integer $m$ with $N \leq m \leq 2 N$ the homogeneous polynomial $p_{m}(z):=\sum_{\xi \in A} h(\xi)\left\langle z, \nu_{\xi}\right\rangle^{m}$ satisfies the following: 
(1) $\left|p_{m}(z)\right|<(1+\theta) h(z)$ for all $z \in \partial \Omega$,

(2) $\left|p_{m}(z)\right|>(1-\theta) h(z)$ for each $z \in \partial \Omega$ such that $\rho(z, \xi) \leq \frac{\theta}{4 \sqrt{c_{2} N}}$ for some $\xi \in A$.

Proof. We can choose $C>2$ and $N_{0} \in \mathbb{N}$ from Lemma 2.3 used for $a=\frac{\theta}{4}$.

Since $h$ has the same values on the circles, there exists $\varepsilon \in(0,1)$ such that we obtain the following property for $z, \xi \in \partial \Omega$ :

$$
c_{1} \rho^{2}(\xi, z)<\varepsilon \Rightarrow \frac{1}{1+a} h(z) \leq h(\xi) \leq \frac{1+2 a}{1+a} h(z) .
$$

Let $\Gamma(z):=\left\{\xi \in A: c_{1} \rho^{2}(\xi, z)<\varepsilon\right\}$. Now let us denote

$$
\begin{aligned}
r_{m}(z) & :=\sum_{\xi \in A \backslash \Gamma(z)} h(\xi)\left\langle z, \nu_{\xi}\right\rangle^{m}, \\
q_{m}(z) & :=\sum_{\xi \in \Gamma(z)} h(\xi)\left\langle z, \nu_{\xi}\right\rangle^{m} .
\end{aligned}
$$

In particular due to Lemma 2.1 we may estimate

$$
\begin{aligned}
\left|r_{m}(z)\right| & \leq\|h\| \sum_{\xi \in A \backslash \Gamma(z)}\left|\left\langle z, \nu_{\xi}\right\rangle\right|^{m} \stackrel{\mathbb{1}}{\leq}\|h\| \sum_{\xi \in A \backslash \Gamma(z)}(1-\varepsilon)^{N} \\
& \stackrel{\mathrm{L} 2.1}{\leq}\|h\| c\left(\frac{C}{2 \sqrt{c_{1} N}}\right)^{1-2 d}(1-\varepsilon)^{N} .
\end{aligned}
$$

Now we may conclude that there exists $N_{1}>N_{0}$ such that for $N_{1} \leq N \leq m \leq 2 N$ and $z \in \partial \Omega$ we have

$$
\left|r_{m}(z)\right|<a \min _{\xi \in \partial \Omega} h(\xi) \leq a h(z) .
$$

Due to Lemma 2.3(4) we may obtain the property (1):

$$
\begin{aligned}
\left|p_{m}(z)\right| & \leq\left|q_{m}(z)\right|+\left|r_{m}(z)\right| \stackrel{\sqrt{3})}{<} \sum_{\xi \in \Gamma(z)} h(\xi)\left|\left\langle z, \nu_{\xi}\right\rangle\right|^{m}+a h(z) \\
& \stackrel{(2)}{<} \frac{1+2 a}{1+a} \sum_{\xi \in A} h(z)\left|\left\langle z, \nu_{\xi}\right\rangle\right|^{m}+a h(z) \stackrel{\mathrm{L} 2.3(4)}{\leq}(1+3 a) h(z) \leq(1+\theta) h(z) .
\end{aligned}
$$

Now let $z \in \partial \Omega$ and $\xi_{0} \in A$ be such that $\rho\left(z, \xi_{0}\right) \leq \frac{a}{\sqrt{c_{2} N}}=\frac{\theta}{4 \sqrt{c_{2} N}}$. Due to Lemma 2.3(3) we may estimate

$$
\begin{aligned}
\left|q_{m}(z)\right| & \geq h\left(\xi_{0}\right)\left|\left\langle z, \nu_{\xi_{0}}\right\rangle\right|^{m}-\sum_{\xi \in \Gamma(z) \backslash\left\{\xi_{0}\right\}} h(\xi)\left|\left\langle z, \nu_{\xi}\right\rangle\right|^{m} \\
& \stackrel{(2)}{\geq} \frac{1}{1+a} h(z)\left|\left\langle z, \nu_{\xi_{0}}\right\rangle\right|^{m}-\sum_{\xi \in \Gamma(z) \backslash\left\{\xi_{0}\right\}} \frac{1+2 a}{1+a} h(z)\left|\left\langle z, \nu_{\xi}\right\rangle\right|^{m} \\
& \stackrel{\text { L2.3(3,1) }}{>} \frac{1-2 a^{2}}{1+a} h(z)-\frac{1+2 a}{1+a} a h(z) \geq \frac{1-a-4 a^{2}}{1+a} h(z) \geq(1-3 a) h(z) .
\end{aligned}
$$

In particular we may obtain property $(2)$ :

$$
\left|p_{m}(z)\right| \geq\left|q_{m}(z)\right|-\left|r_{m}(z)\right|>(1-3 a) h(z)-a h(z) \geq(1-\theta) h(z) .
$$

We improve the above lemma to get a more useful tool in applications. 
Theorem 2.5. For a given constant a $\in(0,1)$ there exists a natural number $K$ such that if $h$ is a continuous strictly positive function on $\partial \Omega$ with the same values on the circles, then there exists $N_{1} \in \mathbb{N}$ such that for all the integers $N$ and $m_{1}, \ldots, m_{K}$ with $N_{1} \leq N \leq m_{1} \leq \ldots \leq m_{K} \leq 2 N$ there exist homogeneous polynomials $p_{m_{1}}, \ldots, p_{m_{K}}$ of a degree $m_{1}, \ldots, m_{K}$ respectively such that $a h(z)<\max _{i=1, \ldots, K}\left|p_{m_{i}}(z)\right|<h(z)$ for all $z \in \partial \Omega$.

Proof. Let $0<c_{1}<c_{2}$ be from (11). For a constant $\theta=\frac{1-a}{1+a}$ we can choose $C, N_{1}$ from Lemma 2.4. Let us consider $N \geq N_{1}$ and denote $A_{N}$ - a maximal $\frac{\theta}{4 \sqrt{c_{2} N}}$-separated subset of $\partial \Omega$. Due to Lemma 2.2 there exists a natural number $K=K\left(\frac{\theta}{4 \sqrt{c_{2}}}, \frac{C}{\sqrt{c_{1}}}\right)$ such that $A_{N}$ can be partitioned into $A_{N}^{1}, \ldots, A_{N}^{K}$ disjoint $\frac{C}{\sqrt{c_{1} N}}$-separated sets.

Now using Lemma 2.4 for a function $h$ and set $A_{N}^{i}$ we may conclude that there exists a homogeneous polynomial $p_{m_{i}}$ of $m_{i}$ degree such that

- $(1+\theta)\left|p_{m_{i}}(z)\right|<(1+\theta) h(z)$ for all $z \in \partial \Omega$.

- $(1+\theta)\left|p_{m_{i}}(z)\right|>(1-\theta) h(z)$ for each $z \in \partial \Omega$ such that $\rho(z, \xi) \leq \frac{\theta}{4 \sqrt{c_{2} N}}$ for some $\xi \in A_{N}^{i}$.

Now let $z \in \partial \Omega$. Since $A_{N}$ is a maximal $\frac{\theta}{4 \sqrt{c_{2} N}}$-separated subset of $\partial \Omega$, there exists $\xi \in A_{N}$ such that $\rho(z, \xi) \leq 1 /\left(4 \sqrt{c_{2} N}\right)$. Due to $A_{N}=\bigcup_{i=1}^{K} A_{N}^{i}$ there exists $i_{0} \in\{1, \ldots, K\}$ such that $\xi \in A_{N}^{i_{0}}$. In particular $\left|p_{m_{i}}(z)\right|>\frac{1-\theta}{1+\theta} h(z)=a h(z)$ and therefore $\max _{i=1, \ldots, K}\left|p_{m_{i}}(z)\right|>a h(z)$, which finishes the proof.

Now we can prove the following fact, which will enable us to construct the bounded holomorphic function with a desired property.

Lemma 2.6. There exists $\theta \in(0,1)$ such that if $\delta_{1}, \delta_{2} \in(0,1), T$ is a compact subset of $\Omega, g$ is a complex continuous function on $\partial \Omega$ and $h$ is a continuous strictly positive function on $\partial \Omega$ such that $|g(z)|<h(z)=h(\lambda z)$ for $|\lambda|=1, z \in \partial \Omega$, then there exists a polynomial $q$ with the following properties:

(1) $\sup _{z \in T}|q(z)|<\delta_{1}$,

(2) $|q(z)|<H(z)$ for all $z \in \partial \Omega$,

(3) $\max _{-\delta_{2} \leq \varphi \leq \delta_{2}}\left|g\left(e^{i \varphi} z\right)+q\left(e^{i \varphi} z\right)\right|>|g(z)|+\theta H(z)$ for $z \in \partial \Omega$,

where $H(z)=h(z)-\max _{|\lambda|=1}|g(\lambda z)|$.

Proof. Let $a=\frac{1}{2}$ and $K$ be a natural number from Theorem 2.5. There exists $\varepsilon \in\left(0, \frac{1}{2} \delta_{2}\right)$ such that $\frac{\pi}{\varepsilon} \in \mathbb{N}$ and $\max _{-2 \varepsilon \leq \varphi \leq 2 \varepsilon}\left|g(z)-g\left(e^{i \varphi} z\right)\right| \leq \frac{H(z)}{8 K}$ for $z \in \partial \Omega$. Let $n=\frac{\pi}{\varepsilon}$. We show that we can choose $\theta=\frac{1}{4 K}$. Due to Theorem 2.5 we conclude that there exists $N_{0} \in \mathbb{N}$ such that for all $m \in \mathbb{N}$ with $N_{0} \leq m n<(m+1) n<\ldots<(m+K) n<2 m n$ there exist homogeneous polynomials $p_{(m+1) n}, \ldots, p_{(m+K) n}$ of $(m+1) n, \ldots,(m+K) n$ degree respectively such that $\frac{1}{2} H(z)<K \max _{j=1, \ldots, K}\left|p_{(m+j) n}(z)\right|<H(z)$ for all $z \in \partial \Omega$. Let us choose $q_{m}:=\sum_{j=1}^{K} p_{(m+j) n}$. We show that it is enough to choose $q=q_{m}$ for $m$ large enough. First we observe that $q_{m}$ fulfills the properties (1)-(2) for $m$ large enough so we only prove property (3). Since $\int_{-\frac{\pi}{n}}^{\frac{\pi}{n}} \exp (-i t k n) d t=0$ for $k \in \mathbb{N}$ we may 
estimate

$$
\begin{aligned}
\sqrt{2 \varepsilon} \max _{-\varepsilon \leq \varphi \leq \varepsilon}\left|q_{m}\left(e^{i \varphi} z\right)\right| & \geq \sqrt{\int_{-\varepsilon}^{\varepsilon}\left|q_{m}\left(e^{i \varphi} z\right)\right|^{2} d \varphi}=\sqrt{\int_{-\frac{\pi}{n}}^{\frac{\pi}{n}} \sum_{j=1}^{K}\left|p_{(m+j) n}(z)\right|^{2} d \varphi} \\
& \geq \sqrt{\frac{2 \pi}{n}} \frac{H(z)}{2 K}=\sqrt{2 \varepsilon} \frac{H(z)}{2 K}
\end{aligned}
$$

for $z \in \partial \Omega$. There exists $\eta_{z} \in[-\varepsilon, \varepsilon]$ such that $\left|q_{m}\left(e^{i \eta_{z}} z\right)\right|=\max _{-\varepsilon \leq \varphi \leq \varepsilon}\left|q_{m}\left(e^{i \varphi} z\right)\right|$. In particular $\left|q_{m}\left(e^{i \eta_{z}} z\right)\right| \geq \frac{H(z)}{2 K}$.

Since

$$
\left|q_{m}\left(e^{i \varphi} z\right)-e^{i m n \varphi} q_{m}(z)\right| \leq \sum_{j=1}^{K}\left|e^{i j n \varphi}-1\right|\left|p_{(m+j) n}(z)\right| \leq \sum_{j=1}^{K}\left|e^{i j n \varphi}-1\right| H(z)
$$

there exists $\varepsilon_{2} \in(0, \varepsilon)$ such that

$$
\left|q_{m}\left(e^{i \varphi} z\right)-e^{i m n \varphi} q_{m}(z)\right|<\frac{H(z)}{8 K}
$$

for $z \in \partial \Omega, m \in \mathbb{N}$ and $-\varepsilon_{2} \leq \varphi \leq \varepsilon_{2}$. Moreover we may observe that if $m n \varepsilon_{2}>\pi$, then there exists $\varphi_{z} \in\left[-\varepsilon_{2}, \varepsilon_{2}\right]$ such that

$$
\left|g(z)+e^{i m n \varphi_{z}} q_{m}\left(e^{i \eta_{z}} z\right)\right|=|g(z)|+\left|q_{m}\left(e^{i \eta_{z}} z\right)\right| .
$$

In particular we may estimate

$$
\begin{aligned}
\max _{-\delta_{2} \leq \varphi \leq \delta_{2}}\left|g\left(e^{i \varphi} z\right)+q_{m}\left(e^{i \varphi} z\right)\right| & \geq \max _{-2 \varepsilon \leq \varphi \leq 2 \varepsilon}\left|g(z)+q_{m}\left(e^{i \varphi} z\right)\right|-\frac{H(z)}{8 K} \\
& \geq \max _{-\varepsilon_{2} \leq \varphi \leq \varepsilon_{2}}\left|g(z)+q_{m}\left(e^{i\left(\eta_{z}+\varphi\right)} z\right)\right|-\frac{H(z)}{8 K} \\
& >\max _{-\varepsilon_{2} \leq \varphi \leq \varepsilon_{2}}\left|g(z)+e^{i m n \varphi} q_{m}\left(e^{i \eta_{z}} z\right)\right|-\frac{H(z)}{4 K} \\
& \geq|g(z)|+\left|q_{m}\left(e^{i \eta_{z}} z\right)\right|-\frac{H(z)}{4 K} \\
& \geq|g(z)|+\frac{H(z)}{4 K} .
\end{aligned}
$$

We have just proved that it is enough to define $q=q_{m}$ for $m$ large enough.

Now we construct an example of a maximum modulus set $K$ which crosses any circle in $\partial \Omega$ with the center at zero. In particular Hausdorff dimension of $K$ is at least $2 d-2$.

Theorem 2.7. Let $\varepsilon>0, T$ be a compact subset of $\Omega, g$ be a complex continuous function on $\partial \Omega$ and $h$ be a positive continuous function on $\partial \Omega$ such that $|g(z)|<$ $h(z)=h(\lambda z)$ for $|\lambda|=1, z \in \partial \Omega$. Then there exists $f \in A(\Omega)$ such that $\|f\|_{T}<\varepsilon$ and $\max _{|\lambda|=1}|(g+f)(\lambda z)|=h(z)$ for $z \in \partial \Omega$.

Proof. Let $\theta \in(0,1)$ be a constant from Lemma 2.6. We construct the sequence of holomorphic polynomials $f_{n} \in A(\Omega)$ with the following properties:

(1) $\left\|f_{n}\right\|_{T}<\frac{\varepsilon}{2^{n+1}}$

(2) $\left|f_{n}\right|<H_{n}$ on $\partial \Omega$,

(3) $\max _{|\lambda|=1}\left|\left(g_{n}+f_{n}\right)(\lambda z)\right|>\left|g_{n}(z)\right|+\theta H_{n}(z)$ for $z \in \partial \Omega$, 
where $g_{1}=g, g_{n+1}=g_{n}+f_{n}, H_{n}(z)=h(z)-\max _{|\lambda|=1}\left|g_{n}(\lambda z)\right|$ for $z \in \partial \Omega$.

To construct $f_{1}$ it is enough to use Lemma 2.6. If $f_{1}, \ldots, f_{n}$ are constructed, we can estimate $\left|g_{n+1}(z)\right|=\left|\left(g_{n}+f_{n}\right)(z)\right| \leq\left|g_{n}(z)\right|+\left|f_{n}(z)\right|<\left|g_{n}(z)\right|+H_{n}(z) \leq h(z)$. Therefore to construct $f_{n+1}$ it suffices to use Lemma 2.6 once again.

Since $H(z)=H(\lambda z)$ for $|\lambda|=1$, due to (3) we can conclude

$$
\max _{|\lambda|=1}\left|\left(g_{n}+f_{n}\right)(\lambda z)\right|>\max _{|\lambda|=1}\left|g_{n}(\lambda z)\right|+\theta H_{n}(z) \geq \theta h(z)+(1-\theta) \max _{|\lambda|=1}\left|g_{n}(\lambda z)\right|,
$$

which implies

$$
\begin{aligned}
H_{n+1}(z) & \leq h(z)-\max _{|\lambda|=1}\left|\left(g_{n}+f_{n}\right)(\lambda z)\right|<(1-\theta)\left(h(z)-\max _{|\lambda|=1}\left|g_{n}(\lambda z)\right|\right) \\
& \leq(1-\theta) H_{n}(z) .
\end{aligned}
$$

The last inequality gives us $\lim _{n \rightarrow \infty} H_{n}(z)=0$. Moreover we can estimate

$$
\sum_{n=m}^{\infty}\left|f_{n}\right|<\sum_{n=m}^{\infty} H_{n}<\sum_{n=m}^{\infty}(1-\theta)^{n} H_{1} \leq(1-\theta)^{m} \frac{\left\|H_{1}\right\|}{\theta}
$$

on $\partial \Omega$. Now we conclude that $f=\sum_{n=1}^{\infty} f_{n} \in A(\Omega)$ and $\max _{|\lambda|=1}|(g+f)(\lambda z)|=$ $h(z)$ for $z \in \partial \Omega$. It is also obvious that $\|f\|_{T}<\varepsilon$.

Let $\sigma$ denote a $(2 d-1)$-dimensional Hausdorff measure on $\partial \Omega$ with $\sigma(\partial \Omega)=1$ and let $h$ be a continuous strictly positive function on $\partial \Omega$ such that $h(z)=h(\lambda z)$ for $|\lambda|=1$. In the paper [3] we constructed a bounded holomorphic function $f_{1}$; i.e. $\left|f_{1}^{*}(z)\right|=h(z)$ for $\sigma$-almost all $z \in \partial \Omega$. Here we construct a new bounded holomorphic function $f_{2}$ with the additional property $\max _{|\lambda|<1}\left|f_{2}(\lambda z)\right|=h(z)$. We use the following fact from the paper [3]:

Lemma ([3, Lemma 3.7]). Let $\varepsilon, \theta \in(0,1), T$ be a compact subset of $\Omega$. If $H$ is a continuous strictly positive function on $\partial \Omega$ and $g$ is a complex continuous function on $\partial \Omega$, then there exists $V$, a relatively open subset of $\partial \Omega$ and $f \in A(\Omega)$ such that

(1) $\|f\|_{T} \leq \varepsilon$,

(2) $|(f+g)(z)|-|g(z)|<H(z)$ for $z \in \partial \Omega$,

(3) $|(f+g)(z)|-|g(z)|>\theta H(z)$ for $z \in V$,

(4) $\sigma(\bar{V})=\sigma(V)>1-2 \varepsilon$.

Theorem 2.8. Let $h$ be a continuous strictly positive function on $\partial \Omega$ such that $h(z)=h(\lambda z)$ for $|\lambda|=1$. Then there exists a bounded holomorphic function $F \in$ $\mathbb{O}(\Omega)$ such that $\left|F^{*}(z)\right|=h(z)$ for almost all $z \in \partial \Omega$ and $\max _{|\lambda|<1}|F(\lambda z)|=h(z)$ for $z \in \partial \Omega$.

Proof. We construct a sequence $\left\{\varepsilon_{m}\right\}_{m \in \mathbb{N}}$ of positive numbers, a sequence $\left\{\omega_{m}\right\}_{m \in \mathbb{N}}$ of holomorphic functions on $\bar{\Omega}$, and a sequence $\left\{V_{m}\right\}_{m \in \mathbb{N}}$ of relatively open subsets of $\partial \Omega$ such that the following properties are fulfilled:

(1) $\omega_{1}=0, \varepsilon_{1}=1,0<4 \varepsilon_{m+1} \leq \varepsilon_{m} \leq 1$,

(2) if $z, w \in \bar{\Omega}$ and $\|z-w\| \leq \varepsilon_{m+1}$, then $\left|\omega_{m}(z)-\omega_{m}(w)\right| \leq \varepsilon_{m}$,

(3) $\left\|\omega_{m+1}-\omega_{m}\right\|_{T_{m}} \leq \varepsilon_{m+1}$ where $T_{m}:=\left\{z \in \bar{\Omega}: \operatorname{dist}(\partial \Omega, z) \geq \varepsilon_{m+1}\right\}$,

(4) $\left(1-2^{-m}\right) h(z)<\max _{|\lambda|=1}\left|\omega_{m+1}(\lambda z)\right|<h(z)$ for $z \in \partial \Omega$,

(5) $\left|\omega_{m+1}\right|-\left|\omega_{m}\right|<H_{m}$ on $\partial \Omega$,

(6) $\left|\omega_{m+1}\right|-\left|\omega_{m}\right|>\left(1-\varepsilon_{m+1}\right) H_{m}$ on $V_{m}$,

(7) $\sigma\left(\bar{V}_{m}\right)=\sigma\left(V_{m}\right)>1-2 \varepsilon_{m+1}$, 
where $H_{1}=h$ and $H_{m+1}:=H_{m}-\left|\omega_{m+1}\right|+\left|\omega_{m}\right|$.

First we construct $\left(\varepsilon_{2}, \omega_{2}, V_{1}\right)$ so that (1)-(7) are fulfilled for $m=1$. Let $\varepsilon_{2} \in(0,0.25)$. Due to Theorem 2.7 there exists $g \in A(\Omega)$ such that $\|g\|_{T_{1}}<\frac{1}{2} \varepsilon_{2}$ and $\max _{|\lambda|=1}|g(\lambda z)|=\frac{3}{4} h(z)$. There exists $\delta \in(0,1)$ so that $|g(z)-g(\delta z)|<$ $\frac{1}{8} \min _{w \in \partial \Omega} h(w)$ for $z \in \partial \Omega$. Now due to [3, Lemma 3.7] there exists $f \in A(\Omega)$ and $V_{1}$, an open subset of $\partial \Omega$ such that

- $\|f\|_{T_{1}}<\frac{1}{2} \varepsilon_{2}$

- $|f(\delta z)|<\frac{1}{8} \min _{w \in \partial \Omega} h(w)$ for $z \in \partial \Omega$,

- $|f+g|-|g|<h-|g|$ on $\partial \Omega$,

- $|f+g|-|g|>\left(1-\varepsilon_{2}\right)(h-|g|)$ on $V_{1}$,

- $\sigma\left(\bar{V}_{1}\right)=\sigma\left(V_{1}\right)>1-2 \varepsilon_{2}$.

Let us choose $\omega_{2}=f+g$. We show that properties (1)-(7) are fulfilled for $m=1$. In fact it is enough to prove only (4) and (6). The remaining properties are trivial. We may estimate

$$
\begin{aligned}
\max _{|\lambda|=1}\left|\omega_{2}(\lambda z)\right| & \geq \max _{|\lambda|=1}|f(\lambda \delta z)+g(\lambda \delta z)| \geq \max _{|\lambda|=1}(|g(\lambda \delta z)|-|f(\lambda \delta z)|) \\
& >\max _{|\lambda|=1}|g(\lambda z)|-\frac{1}{8} \min _{w \in \partial \Omega} h(w)-\frac{1}{8} \min _{w \in \partial \Omega} h(w) \geq \frac{1}{2} h(z)
\end{aligned}
$$

for $z \in \partial \Omega$, which implies (4). Moreover we have

$$
\left|\omega_{2}\right|>|g|+\left(1-\varepsilon_{2}\right)(h-|g|) \geq\left(1-\varepsilon_{2}\right) h+\varepsilon_{2}|g| \geq\left(1-\varepsilon_{2}\right) h
$$

on $V_{1}$, which gives (6).

Now suppose that we have constructed $\varepsilon_{2} \ldots \varepsilon_{m}, \omega_{2} \ldots \omega_{m}, V_{1} \ldots V_{m-1}$. Let us observe that $H_{m}>0$ and

$$
h-H_{m}=\sum_{k=1}^{m-1}\left(H_{k}-H_{k+1}\right)=\sum_{k=1}^{m-1}\left(\left|\omega_{k+1}\right|-\left|\omega_{k}\right|\right)=\left|\omega_{m}\right| .
$$

In particular there exists $\varepsilon_{m+1}$ so that (1)-(2) are fulfilled. Since $\left|\omega_{m}\right|<h$, due to Theorem 2.7 there exists $\widetilde{g} \in A(\Omega)$ such that $\|\widetilde{g}\|_{T_{m}}<\frac{1}{2} \varepsilon_{m+1}$ and $\left(1-2^{-m-1}\right) h(z)<$ $\max _{|\lambda|=1}\left|\widetilde{g}(\lambda z)+\omega_{m}(\lambda z)\right|<h(z)$ for $z \in \partial \Omega$. There exists $\widetilde{\delta} \in(0,1)$ so that

$$
\begin{aligned}
\left(1-2^{-m-1}\right) h(z) & <\max _{|\lambda|=1}\left|\left(\widetilde{g}+\omega_{m}\right)(\widetilde{\delta} \lambda z)\right|, \\
\left(1-\varepsilon_{m+1}\right) H_{m}(z) & <\widetilde{\delta} H_{m}(z)-(1-\widetilde{\delta})\left|\omega_{m}(z)\right|
\end{aligned}
$$

for $z \in \partial \Omega$. Now due to [3, Lemma 3.7] there exists $\tilde{f} \in A(\Omega)$ and $V_{m}$ an open subset of $\partial \Omega$ such that

- $\|\tilde{f}\|_{T_{m}}<\frac{1}{2} \varepsilon_{m+1}$,

- $\max _{|\lambda|=1}|\widetilde{f}(\widetilde{\delta} \lambda z)|<2^{-m-1} h(z)$ for $z \in \partial \Omega$,

- $\left|\widetilde{f}+\widetilde{g}+\omega_{m}\right|-\left|\widetilde{g}+\omega_{m}\right|<h-\left|\widetilde{g}+\omega_{m}\right|$ on $\partial \Omega$,

- $\left|\widetilde{f}+\widetilde{g}+\omega_{m}\right|-\left|\widetilde{g}+\omega_{m}\right|>\widetilde{\delta}\left(h-\left|\widetilde{g}+\omega_{m}\right|\right)$ on $V_{m}$,

- $\sigma\left(\bar{V}_{m}\right)=\sigma\left(V_{m}\right)>1-2 \varepsilon_{m+1}$. 
We show that it is enough to choose $\omega_{m+1}=\widetilde{f}+\widetilde{g}+\omega_{m}$. In fact it suffices to prove the properties (4), (5), and (6). We may estimate

$$
\begin{aligned}
h(z) & >\max _{|\lambda|=1}\left|\omega_{m+1}(\lambda z)\right| \geq \max _{|\lambda|=1}\left|\omega_{m+1}(\widetilde{\delta} \lambda z)\right|>\max _{|\lambda|=1}\left|\left(\widetilde{g}+\omega_{m}\right)(\widetilde{\delta} \lambda z)\right|-2^{-m-1} h(z) \\
& \geq\left(1-2^{-m-1}\right) h(z)-2^{-m-1} h(z)>\left(1-2^{-m}\right) h(z)
\end{aligned}
$$

for $z \in \partial \Omega$, which gives (4). We now prove (5):

$$
\left|\omega_{m+1}\right|-\left|\omega_{m}\right|<h-\left|\omega_{m}\right|=H_{m}+\left|\omega_{m}\right|-\left|\omega_{m}\right|=H_{m} .
$$

To prove (6) we may estimate on $V_{m}$ in the following way:

$$
\begin{aligned}
\left|\omega_{m+1}\right|-\left|\omega_{m}\right| \geq & \left|\omega_{m+1}\right|-\left|\widetilde{g}+\omega_{m}\right|+\left|\widetilde{g}+\omega_{m}\right|-\left|\omega_{m}\right|>\widetilde{\delta}\left(h-\left|\widetilde{g}+\omega_{m}\right|\right) \\
& +\left|\widetilde{g}+\omega_{m}\right|-\left|\omega_{m}\right| \geq \widetilde{\delta} h-\left|\omega_{m}\right| \\
\geq & \widetilde{\delta} H_{m}-(1-\widetilde{\delta})\left|\omega_{m}\right|>\left(1-\varepsilon_{m+1}\right) H_{m} .
\end{aligned}
$$

We can start to construct a bounded holomorphic function. Property (2) implies that $\left\{\omega_{m}\right\}_{m \in \mathbb{N}}$ is a Cauchy sequence, so there exists a function $F \in \mathbb{O}(\Omega)$ such that $\omega_{m} \rightarrow F$ uniformly on the compact subsets of $\Omega$.

We prove the maximum property of $F$. Since $\left|\omega_{m+1}\right|<H_{m}+\left|\omega_{m}\right|=h$ we can conclude $|F| \leq h$ on $\partial \Omega$. Moreover using the properties (2), (3), and (4) we may conclude that

$$
\begin{aligned}
\max _{|\lambda|<1}\left|F\left(\left(1-\varepsilon_{m+1}\right) \lambda z\right)\right| & \geq \max _{|\lambda|<1}\left|\omega_{m}\left(\left(1-\varepsilon_{m+1}\right) \lambda z\right)\right|-\sum_{k=m}^{\infty}\left\|\omega_{k}-\omega_{k+1}\right\|_{T_{m}} \\
& \geq \max _{|\lambda|<1}\left|\omega_{m}\left(\left(1-\varepsilon_{m+1}\right) \lambda z\right)\right|-\sum_{k=m}^{\infty} \varepsilon_{k+1} \\
& \geq \max _{|\lambda|<1}\left|\omega_{m}(\lambda z)\right|-\varepsilon_{m}-\varepsilon_{m} \sum_{k=1}^{\infty} 4^{-k} \\
& >\left(1-2^{-m+1}\right) h(z)-2 \varepsilon_{m}
\end{aligned}
$$

for $z \in \partial \Omega$. In particular

$$
h(z) \geq \max _{|\lambda|<1}|F(\lambda z)| \geq \lim _{m \rightarrow \infty} \max _{|\lambda|<1}\left|F\left(\left(1-\varepsilon_{m+1}\right) \lambda z\right)\right|=h(z)
$$

for $z \in \partial \Omega$.

Let $D_{k}:=\bigcap_{m=k}^{\infty} V_{m}$ and $D=\bigcup_{k=1}^{\infty} D_{k}$. Let us observe that

$$
\sigma\left(D_{k}\right) \geq 1-2 \sum_{n=k}^{\infty} \varepsilon_{n} \geq 1-2 \varepsilon_{k-1} \sum_{n=1}^{\infty} 4^{-n}>1-\varepsilon_{k-1} .
$$

In particular $\sigma(D)=1$.

Let $z \in D$. Then there exists $m_{z} \in \mathbb{N}$ such that $z \in V_{m}$ for $m \geq m_{z}$. Since $h=H_{m}+\left|\omega_{m}\right|$ we may estimate

$$
\begin{aligned}
\left|F\left(\left(1-\varepsilon_{m+2}\right) z\right)\right| & \geq\left|\omega_{m+1}\left(\left(1-\varepsilon_{m+2}\right) z\right)\right|-\sum_{k=m+1}^{\infty}\left\|\omega_{k}-\omega_{k+1}\right\|_{T_{m+1}} \\
& \geq\left|\omega_{m+1}(z)\right|-2 \varepsilon_{m+1}>\left|\omega_{m}(z)\right|+\left(1-\varepsilon_{m+1}\right) H_{m}(z)-2 \varepsilon_{m+1} \\
& \geq\left(1-\varepsilon_{m+1}\right) h(z)+\varepsilon_{m+1}\left|\omega_{m}(z)\right|-2 \varepsilon_{m+1} \\
& \geq\left(1-\varepsilon_{m+1}\right) h(z)-2 \varepsilon_{m+1}
\end{aligned}
$$


for $z \in V_{m}$. In particular $\lim _{m \rightarrow \infty}\left|F\left(\left(1-\varepsilon_{m+1}\right) z\right)\right|=h(z)$ for $z \in D$, which implies that $\left|F^{*}(z)\right|=h(z)$ for almost all $z \in \partial \Omega$.

\section{REFERENCES}

[1] A.B. Aleksandrov: The existence of inner functions in a ball, Math. Sb. 118(160), 147-163 (1982). MR658785 (83i:32002)

[2] P. Kot: Homogeneous polynomials on strictly convex domains, Proc. Amer. Math. Soc. 135, 3895-3903 (2007). MR2341939

[3] P. Kot: A holomorphic function with given almost all boundary values on a domain with holomorphic support function, Journal of Convex Analysis 14, no. 4, 693-704 (2007). MR2350811

[4] E. Løw: Inner functions and boundary values in $H^{\infty}(\Omega)$ and $A(\Omega)$ in smoothly bounded pseudoconvex domains, Math. Z. 185, 191-210 (1984). MR731340(85d:32032)

[5] B.S. Henriksen: A peak set of Hausdorff dimension $2 n-1$ for the algebra $A(D)$ in the boundary of a domain $D$ with $C^{\infty}$-boundary in $\mathbb{C}^{n}$, Math. Ann. 259, 271-277 (1982). MR656666 (83k:32028)

[6] E.L. Stout: The dimension of peak-interpolation sets, Proc. Amer. Math. Soc. 86, no. 3, 413-416 (1982). MR671206 (84e:32015)

[7] E.L. Stout, Th. Duchamp: Maximum modulus sets, Ann. Inst. Fourier (Grenoble) 31, no. 3, 37-69 (1981). MR638616(83d:32019)

Politechnika Krakowska, Instytut Matematyki, ul. Warszawska 24, 31-155 Kraków, POLAND

E-mail address: pkot@pk.edu.pl 